\title{
Moments and associated measures of copulas with fractal support
}

\author{
Enrique de $\mathrm{Amo}^{1}$ \\ University of Almería, Spain \\ Manuel Díaz Carrillo \\ University of Granada, Spain \\ Juan Fernández Sánchez \\ University of Almería, Spain \\ Antonio Salmerón \\ University of Almería, Spain
}

\begin{abstract}
Copulas are closely related to the study of distributions and the dependence between random variables. In this paper we develop a recurrence formula for the moments of a measure associated with a copula (a bivariate distribution function with uniform one-dimensional marginals) in the case that its support is a fractal set. We do the same for its principal and secondary diagonals. We also study certain measures of dependence or association for these copulas with fractal supports.
\end{abstract}

Keywords: Moment, copula, distribution function 2010 MSC: 28D05, 60E05, 62H20

Email addresses: edeamo@ual.es (Enrique de Amo), madiaz@ugr.es (Manuel Díaz Carrillo), juanfernandez@ual .es (Juan Fernández Sánchez), antonio.salmeron@ual.es (Antonio Salmerón)

${ }^{1}$ Corresponding author. 


\section{Introduction}

Copulas are mathematical objects which started to be studied in depth just a few years ago. Since Sklar proved his celebrated theorem in 1959, the study of copulas and their applications has revealed itself as a tool of great interest in several branches of mathematics. For an introduction to copulas see the book by Nelsen [15].

In the literature we have examined, all the examples of singular copulas (see (4) below) we have found, are supported by sets with Hausdorff dimension 1. However, it is implied in some papers (e.g. [19]) that the well-known examples of Peano and Hilbert curves, originate self-affine copulas with selfaffine fractal support, since the Hausdorff dimension of their graphs is $3 / 2$ (see $[14,22])$.

Recently, Fredricks et al. [6], using an iterated function system, constructed families of copulas whose supports are fractals. In particular, they give sufficient conditions for the support of a self-similar copula to be a fractal whose Hausdorff dimension is between 1 and 2. The study of these functions has continued in [1].

It is well known that for each copula $C$ there exists an associated measure $\mu_{C}$ that is doubly stochastic (see (2) below). In the case in which the support $S$ of the copula $C$ is a fractal set, the computations related with the calculus of the integral of functions with respect to the measure $\mu_{C}$ are rather complicated. Among the more interesting integrals to be computed, we find the moments and the concordance or associated measures.

Let us recall that for a finite and positive measure $\mu$, it is possible to give a representation of complex polynomials

$$
p_{n}: \mathbb{C} \longrightarrow \mathbb{C}
$$

where $p_{n}(z)=p_{n}(z, \mu)=\kappa_{n} z^{n}+\cdots+\kappa_{1} z+\kappa_{0}$, with $\kappa_{n}>0$, in such way that they constitute an orthonormal system in $L^{2}(\mu)$, that is

$$
\int p_{m} \bar{p}_{n} d \mu=\left\{\begin{array}{ll}
0, & \text { if } m \neq n \\
1, & \text { if } m=n
\end{array} .\right.
$$

These polynomials can be determined by the complex moments of the measure; they are the numbers $\sigma_{i, j}=\int z^{i} \overline{z^{j}} d \mu(z)$.

Therefore, the orthonormal polynomials are determined by the moments $\iint x^{m} y^{n} d \mu_{C}(x, y)$ (see, for example, [21] and [20]). 
On the other hand, integral calculus for functions is required to study association measures for copulas. Again, computations are complicated when the support of the copula is a fractal set.

In this paper we tackle these two problems in the context of the copulas with fractal support introduced by Fredricks et al. [6]. Specifically, in Section 3 , we present a recurrence formula that gives rise to the real moments for measures that are associated to copulas. Likewise, we do this for the (primary) diagonal section and for the secondary (or opposite) diagonal section of a copula. We provide algorithms to simplify computations on some of these cases.

Moreover, we also provide several examples of the moments and polynomials for particular cases.

Section 4 is devoted to the study of concordance measures for these copulas.

Although our study is restricted to the family of copulas with fractal support with Hausdorff dimension $s \in$ ]1,2[, given by Fredricks et al. in [6], the techniques we present to calculate moments and measures of association can be applied to the study of any self-similar copula. In this general case, it is also possible to obtain similar recurrent formulas to those in Section 3, and to calculate measures of association as we have done in Section 4.

\section{Preliminaries}

This section contains background information.

(1) Let $\mathbb{I}$ be the closed unit interval $[0,1]$ and let $\mathbb{I}^{2}=\mathbb{I} \times \mathbb{I}$ be the unit square. A two-dimensional copula (or a copula, for brevity) $C: \mathbb{I}^{2} \longrightarrow \mathbb{I}$ is the restriction to $\mathbb{I}^{2}$ of a bivariate distribution function whose univariate marginals are uniformly distributed on $\mathbb{I}$. Each copula $C$ induces a probability measure $\mu_{C}$ on $\mathbb{I}^{2}$ via the formula

$$
\mu_{C}([a, b] \times[c, d])=C(b, d)-C(b, c)-C(a, d)+C(a, c),
$$

in a similar fashion to joint distribution functions; that is, the $\mu_{C}$-measure of a set is its $C$-volume (that is, the probability mass). Through standard measure-theoretical techniques, $\mu_{C}$ can be extended from the semi-ring of rectangles in $\mathbb{I}^{2}$ to the $\sigma$-algebra $\mathcal{B}\left(\mathbb{I}^{2}\right)$ of the Borel sets. The measure $\mu_{C}$ is doubly stochastic. For an introduction to copulas see [15].

(2) For our further consideration, we remark that Sklar's theorem makes the following statement: If $C$ is a copula and $F$ and $G$ are distribution 
functions, then the function $H$, defined by $H(x, y)=C(F(x), G(y))$, is a joint distribution functions with marginals $F$ and $G$.

(3) A transformation matrix $T$ is a matrix with non-negative entries, for which the sum of all the entries is 1 and neither the row nor the column sums of entries are zero.

Following the paper by Fredricks et al. [6], we recall that each transformation matrix $T$ determines a subdivision of $\mathbb{I}^{2}$ into subrectangles $R_{i j}=$ $\left[p_{i-1}, p_{i}\right] \times\left[q_{j-1}, q_{j}\right]$, where $p_{i}$ (respect., $q_{j}$ ) denotes the sum of the entries in the first $i$ columns (respect., $j$ rows) of $T$. For a transformation matrix $T$ and a copula $C, T(C)$ denotes the copula that, for each $(i, j)$, concentrates its mass on $R_{i j}$ in the same way in which $C$ concentrates its mass on $\mathbb{I}^{2}$.

Theorem 2 in [6] shows that for each transformation matrix $T \neq[1]$, there is a unique copula $C_{T}$ for which $T\left(C_{T}\right)=C_{T}$.

(4) Let $T$ be a transformation matrix. We now consider the following conditions for $T$ :

i) $T$ has, at least, one zero entry.

ii) For each non-zero entry of $T$, the row and column sums through that entry are equal.

iii) There is, at least, one row or column of $T$ with two non-zero entries.

Theorem 3 in [6], shows that if $T$ is a transformation matrix with i) in (4), then $C_{T}$ is singular (that is, its support has either zero Lebesgue measure or $\left.\mu_{C_{T}} \equiv \mu_{C_{T}}^{s}\right)$.

We say that a copula $C$ is invariant if $C=C_{T}$ for some transformation matrix $T$. An invariant copula $C_{T}$ is said to be self-similar if $T$ satisfies ii) in (4).

Theorem 6 in [6] shows that the support of a self-similar copula $C_{T}$ for which $T$ satisfies i) and iii) in (4), is a fractal with Hausdorff dimension between 1 and 2 .

(5) Finally, a mapping $f: \mathbb{R}^{n} \longrightarrow \mathbb{R}^{n}$ is called a contracting similarity (or a similarity transformation of ratio $r$ ) if there is $r, 0<r<1$, such that $\|f(x)-f(y)\|=r\|x-y\|$, for all $x, y \in \mathbb{R}^{n}$. A similarity transforms subsets of $\mathbb{R}^{n}$ into geometrically similar sets. The invariant set (or attractor) for a finite family of similitaries is said to be a self-similar set. Theorem 4 in [6] shows that the support of the copula $C_{T}$ is the invariant set for a system of similarities obtained from the partitions of $\mathbb{I}^{2}$ determined by $T$ (see (3) above). For an introduction to the techniques of representation of some fractals via iterated function systems, see $[7,8]$. 


\section{A recurrence formula for the moments}

In the last decades several papers have reported on the study of moments and their asymptotic values for singular distributions, motivated by the fact that the coefficients of the orthogonal polynomials with respect to a distribution are actually determined by these moments (see for example $[3,10,11,13])$.

In this section we give two recurrence formulas to compute the moments of copulas with fractal support.

The main tools we use are self-similarity and the relation between a characteristic function and the moments of the distribution.

\subsection{First method}

We note that a copula $C$ can be considered as a particular case of a bivariate distribution function (see (1)). Then, for $C$ we have that its characteristic function is defined by

$$
\phi\left(t_{1}, t_{2}\right)=\int_{\mathbb{I}^{2}} e^{\left(x t_{1}+y t_{2}\right) i} d \mu_{C}(x, y), \quad\left(t_{1}, t_{2}\right) \in \mathbb{C}^{2}
$$

and the moments are defined as (see [4, Sec. 26])

$$
M_{m, n}=\int_{\mathbb{I}^{2}} x^{m} y^{n} d \mu_{C}(x, y) .
$$

Now, we recall that the notion of an iterated function system (IFS) may be extended to define invariant measures supported by the attractor of the system.

Definition 3.1. Let $\left\{F_{1}, \ldots, F_{m}\right\}$ be an IFS on $K \subset \mathbb{R}^{n}$ and $p_{1}, \ldots, p_{m}$ be "probabilities" or "mass ratios", with $p_{i}>0$ for all $i$ and $\sum_{i=1}^{m} p_{i}=1$. A measure $\mu$ is said to be self-similar if $\mu(A)=\sum_{i=1}^{m} p_{i} \mu\left(F_{i}^{-1}(A)\right)$ for any Borel set $A$.

The existence of such a measure is ensured by $[9$, Th. 2.8] or $[12, \S$ 4.4]. We introduce the following result for computational purposes (see (5) above): 
Lemma 3.2. Let $K \subset \mathbb{R}^{n}$ and let $\mu$ be a self-similar measure associated with the family of similarity transformations $\left\{F_{1}, \ldots, F_{m}\right\}$ with respective mass ratios $\left\{p_{1}, \ldots, p_{m}\right\}$. Then, for any continuous function $g: K \rightarrow \mathbb{R}$ and any $k,(1 \leq k \leq m)$, we have

$$
\int_{F_{k}(K)} g(x) d \mu(x)=p_{k} \int_{K} g\left(F_{k}(x)\right) d \mu(x) .
$$

Proof. The map $F_{k}$ is a self-similarity transformation, therefore, it is an isomorphism between measurables spaces. As a consequence, there exists a natural bijection from the step functions on $K$ to $F_{k}(K)$ (considering the induced $\sigma$-algebra, in both cases). The measures of the measurable sets $A$ and $F_{k}(A)$ are proportional to ratio $p_{k}$, therefore, and the statement is true in the case that $g$ is a step function. Density arguments establish that the statement is also true for all integrable functions.

(6) An immediate consequence derived from the above lemma is the following useful expression:

$$
\int_{K} g(x) d \mu(x)=\sum_{k=1}^{m} p_{k} \int_{K} g\left(F_{k}(x)\right) d \mu(x) .
$$

Now, we consider the family of transformation matrices

$$
T_{r}=\left(\begin{array}{ccc}
r / 2 & 0 & r / 2 \\
0 & 1-2 r & 0 \\
r / 2 & 0 & r / 2
\end{array}\right)
$$

with $r \in] 0, \frac{1}{2}\left[\right.$. According to (3) and (4) above, $\left\{C_{r}=C_{T_{r}}: r \in\right] 0, \frac{1}{2}[\}$ is a family of copulas supported by a fractal with Hausdorff dimension in the interval $] 1,2[$.

In the following result, we apply (6) to the copulas $C_{T_{r}}$.

Proposition 3.3 (Functional Equation). The characteristic function of $C_{T_{r}}$, which we denote by $c_{r}$, satisfies:

$$
\begin{aligned}
c_{r}\left(t_{1}, t_{2}\right)= & \frac{r}{2}\left(1+e^{i(1-r) t_{1}}+e^{i(1-r) t_{2}}+e^{i(1-r)\left(t_{1}+t_{2}\right)}\right) c_{r}\left(r t_{1}, r t_{2}\right)+ \\
& +(1-2 r) e^{i r\left(t_{1}+t_{2}\right)} c_{r}\left((1-2 r) t_{1},(1-2 r) t_{2}\right) .
\end{aligned}
$$


Proof. A rewriting of (6) is sufficient when we take into account the weights of the matrix, and the particular form of the involved function $g$.

This equation has a case of special interest. If $r=1 / 3$, then the characteristic function is given by an infinite convolution:

Corollary 3.4. If $r=1 / 3$, then:

$$
c_{1 / 3}\left(t_{1}, t_{2}\right)=\prod_{k=1}^{\infty}\left(\frac{1}{6}\left(1+e^{i \frac{2}{3^{k}} t_{1}}+e^{i \frac{2}{3^{k}} t_{2}}+e^{i \frac{2}{3^{k}}\left(t_{1}+t_{2}\right)}\right)+\frac{1}{3} e^{i \frac{1}{3^{k}}\left(t_{1}+t_{2}\right)}\right) .
$$

Therefore, the probability associated with the copula is given by an infinite convolution of probabilities $p_{k}$ satisfying:

$$
\left\{\begin{array}{l}
p_{k}((0,0))=p_{k}\left(\left(0, \frac{2^{k}}{3^{k}}\right)\right)=p_{k}\left(\left(\frac{2^{k}}{3^{k}}, 0\right)\right)=p_{k}\left(\left(\frac{2^{k}}{3^{k}}, \frac{2^{k}}{3^{k}}\right)\right)=\frac{1}{6} \\
p_{k}\left(\left(\frac{1}{3^{k}}, \frac{1}{3^{k}}\right)\right)=1 / 3 .
\end{array}\right.
$$

Proof. The complex function in two complex variables

$$
H\left(\left(t_{1}, t_{2}\right)\right)=\prod_{k=1}^{\infty}\left(\frac{1}{6}\left(1+e^{i \frac{2}{3^{k}} t_{1}}+e^{i \frac{2}{3^{k}} t_{2}}+e^{i \frac{2}{3^{k}}\left(t_{1}+t_{2}\right)}\right)+\frac{1}{3} e^{i \frac{1}{3^{k}}\left(t_{1}+t_{2}\right)}\right)
$$

is holomorphic (see for example [5]). If $r=1 / 3$, then:

$$
c_{1 / 3}\left(t_{1}, t_{2}\right)=\left(\frac{1}{6}\left(1+e^{i \frac{2}{3} t_{1}}+e^{i \frac{2}{3} t_{2}}+e^{i \frac{2}{3}\left(t_{1}+t_{2}\right)}\right)+\frac{1}{3} e^{i \frac{1}{3}\left(t_{1}+t_{2}\right)}\right) c_{1 / 3}\left(\frac{t_{1}}{3}, \frac{t_{2}}{3}\right) .
$$

In general, we have that:

$$
c_{1 / 3}\left(\frac{t_{1}}{3^{n+1}}, \frac{t_{2}}{3^{n+1}}\right)=\frac{c_{1 / 3}\left(t_{1}, t_{2}\right)}{\prod_{k=1}^{n}\left(\frac{1}{6}\left(1+e^{i \frac{2}{3^{k}} t_{1}}+e^{i \frac{2}{3^{k}} t_{2}}+e^{i \frac{2}{3^{k}}\left(t_{1}+t_{2}\right)}\right)+\frac{1}{3} e^{i \frac{1}{3^{k}}\left(t_{1}+t_{2}\right)}\right)} ;
$$

and the continuity of $c_{1 / 3}$ implies that:

$$
\frac{c_{1 / 3}\left(t_{1}, t_{2}\right)}{H\left(\left(t_{1}, t_{2}\right)\right)}=c_{1 / 3}(0,0)=1
$$


Corollary 3.5. The moments for copula $C_{T_{r}}$ satisfy the relation:

$$
M_{m, n}=m ! n ! \frac{A+B+C+D}{1-2 r^{m+n+1}-(1-2 r)^{m+n+1}},
$$

where

$$
\left\{\begin{array}{l}
A=\frac{r}{2} \sum_{a=1}^{m} \frac{(1-r)^{a} r^{m+n-a} M_{m-a, n}}{a ! n !(m-a) !} \\
B=\frac{r}{2} \sum_{b=1}^{m} \frac{(1-r)^{b} r^{m+n-b} M_{m, n-b}}{b ! m !(n-b) !} \\
C=\frac{r}{2} \sum_{a=0}^{m} \sum_{b=0}^{n *} \frac{(1-r)^{a+b} r^{m+n-a-b} M_{m-a, n-b}}{a ! b !(m-a) !(n-b) !} \\
D=(1-2 r) \sum_{a=0}^{m} \sum_{b=0}^{n *} \frac{r^{a+b}(1-2 r)^{m+n-a-b} M_{m-a, n-b}}{a ! b !(m-a) !(n-b) !} .
\end{array}\right.
$$

(The asterisks mean that the sums run through the whole range, but $a=b=$ 0.)

Proof. We use the series expansion to substitute the exponential function in the integral

$$
\int_{\mathbb{I}^{2}} e^{\left(x t_{1}+y t_{2}\right) i} d \mu_{C_{r}}(x, y) .
$$

Now, by the Monotone Convergence Theorem we can commute series and integral:

$$
\begin{aligned}
c_{r}\left(t_{1}, t_{2}\right) & =\int_{[0,1]^{2}} e^{\left(x t_{1}+y t_{2}\right) i} d \mu_{C_{r}}(x, y)=\sum_{k=0}^{\infty} \sum_{n+m=k} \frac{i^{m+n}}{m ! n !} M_{m, n} t_{1}^{m} t_{2}^{n} \\
& =\sum_{m=0}^{\infty} \sum_{n=0}^{\infty} \frac{i^{m+n}}{m ! n !} M_{m, n} t_{1}^{m} t_{2}^{n} .
\end{aligned}
$$

To apply the functional equation, we substitute it in the equation above, obtaining

$$
\left\{\begin{array}{l}
c_{r}\left(r t_{1}, r t_{2}\right)=\sum_{m=0}^{\infty} \sum_{n=0}^{\infty} \frac{i^{m+n} r^{m+n}}{m ! n !} M_{m, n} t_{1}^{m} t_{2}^{n}, \\
c_{r}\left((1-2 r) t_{1},(1-2 r) t_{2}\right)=\sum_{m=0}^{\infty} \sum_{n=0}^{\infty} \frac{i^{m+n}(1-2 r)^{m+n}}{m ! n !} M_{m, n} t_{1}^{m} t_{2}^{n}
\end{array}\right.
$$

Now, if we multiply this series by the series of $1+e^{i(1-r) t_{1}}+e^{i(1-r) t_{2}}+$ 
$e^{i(1-r)\left(t_{1}+t_{2}\right)}$ and $(1-2 r) e^{i r\left(t_{1}+t_{2}\right)}$, then we obtain the equality:

$$
\begin{aligned}
& \sum_{m=0}^{\infty} \sum_{n=0}^{\infty} \frac{i^{m+n}}{m ! n !} M_{m, n} t_{1}^{m} t_{2}^{n} \\
= & \sum_{m=0}^{\infty} \sum_{n=0}^{\infty} \frac{r}{2} \frac{i^{m+n}}{m ! n !} M_{m, n} t_{1}^{m} t_{2}^{n}+ \\
& +\sum_{m=0}^{\infty} \sum_{n=0}^{\infty} \frac{r}{2} \sum_{a=0}^{m} \frac{i^{m+n}(1-r)^{a} r^{m+n-a} M_{m-a, n}}{a ! n !(m-a) !} t_{1}^{m} t_{2}^{n}+ \\
& +\sum_{m=0}^{\infty} \sum_{n=0}^{\infty} \frac{r}{2} \sum_{b=0}^{m} \frac{i^{m+n}(1-r)^{b} r^{m+n-b} M_{m, n-b}}{b ! m !(n-b) !} t_{1}^{m} t_{2}^{n}+ \\
& +\sum_{m=0}^{\infty} \sum_{n=0}^{\infty} \frac{r}{2} \sum_{a=0}^{m} \sum_{b=0}^{n} \frac{i^{m+n}(1-r)^{a+b} r^{m+n-a-b} M_{m-a, n-b}}{a ! b !(m-a) !(n-b) !} t_{1}^{m} t_{2}^{n}+ \\
& +\sum_{m=0}^{\infty} \sum_{n=0}^{\infty}(1-2 r) \sum_{a=0}^{m} \sum_{b=0}^{n} \frac{i^{m+n} r^{a+b}(1-2 r)^{m+n-a-b} M_{m-a, n-b}}{a ! b !(m-a) !(n-b) !} t_{1}^{m} t_{2}^{n} .
\end{aligned}
$$

Finally, doing equalities in exponent at $t_{1}^{m} t_{2}^{n}$, and working out the value $M_{m, n}$, we deduce the equality in the statement.

\subsection{Second method}

Another way to prove the formula for the moments can be obtained via the integral of $x^{m} y^{n}$.

Proof. Now, the application of (6) gives rise to:

$$
\begin{aligned}
\int_{\mathbb{I}^{2}} x^{m} y^{n} d \mu_{C_{r}}= & \frac{r}{2} \int_{\mathbb{I}^{2}}(r x)^{m}(r y)^{n} d \mu_{C_{r}}+\frac{r}{2} \int_{\mathbb{I}^{2}}(r x+1-r)^{m}(r y)^{n} d \mu_{C_{r}} \\
& +\frac{r}{2} \int_{\mathbb{I}^{2}}(r x)^{m}(r y+1-r)^{n} d \mu_{C_{r}} \\
& +\frac{r}{2} \int_{\mathbb{I}^{2}}(r x+1-r)^{m}(r y+1-r)^{n} d \mu_{C_{r}} \\
& +(1-2 r) \int_{\mathbb{I}^{2}}((1-2 r) x+r)^{m}((1-2 r) y+r)^{n} d \mu_{C_{r}} .
\end{aligned}
$$


Let us compute into the integral:

$$
\begin{aligned}
M_{m, n}= & \frac{r^{1+n+m}}{2} M_{m, n}+\frac{r^{1+n}}{2} \sum_{a=0}^{m}\left(\begin{array}{c}
m \\
a
\end{array}\right) r^{a}(1-r)^{m-a} M_{a, n} \\
& +\frac{r^{1+m}}{2} \sum_{b=0}^{n-1}\left(\begin{array}{c}
n \\
b
\end{array}\right) r^{b}(1-r)^{n-b} M_{m, b} \\
& +\frac{r}{2} \sum_{a=0}^{n} \sum_{b=0}^{m}\left(\begin{array}{c}
m \\
a
\end{array}\right)\left(\begin{array}{c}
n \\
b
\end{array}\right) r^{a+b}(1-r)^{n+m-a-b} M_{a, b} \\
& +(1-2 r) \sum_{a=0}^{n} \sum_{b=0}^{m}\left(\begin{array}{c}
m \\
a
\end{array}\right)\left(\begin{array}{l}
n \\
b
\end{array}\right)(1-2 r)^{a+b} r^{n+m-a-b} M_{a, b} .
\end{aligned}
$$

Finally, if we work out $M_{m, n}$, then we state the result.

\subsection{Diagonal sections}

For a given copula $C$, there are two distinguishing functions $\delta_{1}, \delta_{2}$ from $\mathbb{I}$ to $\mathbb{I}$ defined by $\delta_{1}(x)=C(x, x)$ (the primary diagonal section, or simply, the diagonal of $C$ ), and $\delta_{2}(x)=C(x, 1-x)$ (the secondary or opposite diagonal section, or simply, the opposite diagonal of $C$ ). In general, the sections of a copula are employed in the construction of copulas, and to provide interpretations of certain dependence properties (see [15]). The diagonal section is an absolutely continuous distribution function; but its moments are rather complicated to compute.

Applying the same arguments as the above sections, and using the next result on functional equations systems (we add the observation that a perturbation exists produced by an absolutely continuous function), it is possible to obtain two recurrence formulas for the moments of $n$-order with respect to the family of copulas $\left\{C_{T_{r}}: 0<r<1 / 2\right\}$.

Proposition 3.6 (Functional Equations). The diagonal section $\delta_{1}$ is the unique function that satisfies the functional equations that follow:

$$
\left\{\begin{array}{l}
\delta_{1}(r x)=\frac{r}{2} \delta_{1}(x) \\
\delta_{1}(r+(1-2 r) x)=\frac{r}{2}+(1-2 r) \delta_{1}(x) \\
\delta_{1}(1-r+r x)=1-\frac{3 r}{2}+\frac{r}{2} \delta_{1}(x)+r x
\end{array}\right.
$$

These functional equations remind us those studied by De Rham in [16]. The De Rham functions have no an easy expression, but we can obtain it 
using representaion systems (see [2]). The term $r x$ in the third equation can be consider as a perturbation that provides absolute continuity to $\delta_{1}$, while the De Rham functions are singular.

Proposition 3.7. The characteristic function of the diagonal section $\delta_{1}$ satisfies the relation:

$$
c(t)=\frac{r}{2}\left(1+e^{i(1-r) t}\right) c(r t)+(1-2 r) e^{i r t} c((1-2 r) t)+r \frac{e^{i t}-e^{i t(1-r)}}{i t} .
$$

Corollary 3.8. In the case that $r=1 / 3$, then:

$$
c(t)=\frac{1}{3 i t} \sum_{k=0}^{\infty} 3^{k}\left(e^{i t / 3^{k}}-e^{2 i t / 3^{k+1}}\right)\left(\frac{1}{6}+\frac{1}{3} e^{i / 3}+\frac{1}{6} e^{2 i / 3}\right)^{k} .
$$

Corollary 3.9. The m-moment for the diagonal section, that is, $M_{m}:=$ $\int_{0}^{1} x^{m} d \delta_{1}(x)$ equals to:

$$
\frac{\sum_{k=0}^{m-1}\left(\begin{array}{c}
m \\
k
\end{array}\right)(1-2 r)^{k+1} r^{m-k} M_{k}+\frac{1}{2} \sum_{k=0}^{m-1}\left(\begin{array}{c}
m \\
k
\end{array}\right)(1-r)^{m-k} r^{k+1} M_{k}+\frac{r^{m+1}+1-(1-r)^{m+1}}{2(m+1)}}{1-r^{m+1}-(1-2 r)^{m+1}} .
$$

We recall that the opposite or secondary diagonal section it is not a distribution function because the function $\delta_{2}$ is not monotone. But it is a bounded variation function and, therefore, it has an associated signed measure, say $d \delta_{2}$. We can study the corresponding moments for this signed measure:

Proposition 3.10 (Functional Equations). The opposite diagonal section satisfies the following system of functional equations:

$$
\left\{\begin{array}{l}
\delta_{2}(r x)=\frac{r}{2} \delta_{2}(x)+\frac{r}{2} x \\
\delta_{2}(r+(1-2 r) x)=\frac{r}{2}+(1-2 r) \delta_{2}(x) \\
\delta_{2}(1-r+r x)=\frac{r}{2} \delta_{2}(x)+\frac{r}{2}(1-x) .
\end{array}\right.
$$

Proposition 3.11. The characteristic function of the opposite diagonal section $\delta_{2}$, that is $\int_{0}^{1} e^{i x} d \delta_{2}(x)$, satisfies the relation:

$c(t)=\frac{r}{2}\left(1+e^{i(1-r)}\right) c(r t)+(1-2 r) e^{i r t} c((1-2 r) t)+\frac{r}{2} \frac{e^{i t r}+e^{i t(1-r)}-1-e}{i t}$. 
Corollary 3.12. The m-moment for the opposite diagonal section, that is, $M_{m}^{*}:=\int_{0}^{1} x^{m} d \delta_{2}(x)$, satisfies the relation:

$M_{m}^{*}=\frac{\sum_{a=0}^{m-1}\left(\begin{array}{c}m \\ a\end{array}\right)(1-2 r)^{a+1} \frac{r^{m-a+1}}{2} M_{a}^{*}+\frac{1}{2} \sum_{a=0}^{m-1}\left(\begin{array}{c}m \\ a\end{array}\right)(1-r)^{m-a+1} r^{a} M_{a}^{*}+\frac{r}{2} \frac{r^{m+1}-1+(1-r)^{m+1}}{(m+1)}}{1-r^{m+1}-(1-2 r)^{m+1}}$.

Example 3.13. We give some examples we obtain using Corollaries 3.5, 3.9 and 3.12 with $r \in] 0,1\left[\right.$. Figure 1 shows the graphs for the moments $M_{2,3}$, $M_{3,3}$ and $M_{3,4}$. The graphs that correspond to the first eight moments of the diagonal section of $C_{r}$ are given in Fig. 2.

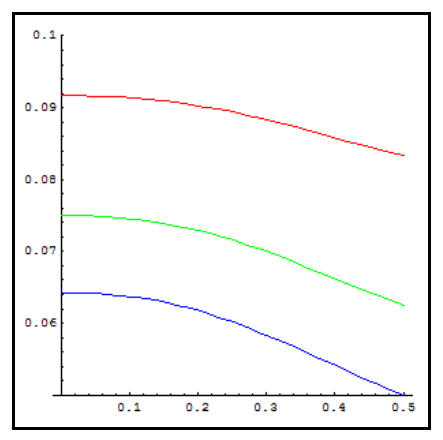

Figure 1: Graphs for the moments $M_{2,3}, M_{3,3}$ and $M_{3,4}$

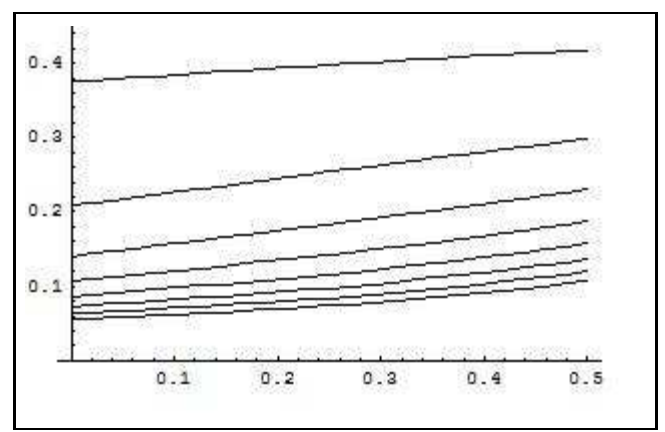

Figure 2: Graphs for the moments of the diagonal section

For the secondary diagonal section, corresponding graphs for the moments $M_{8}^{*}$ to $M_{1}^{*}$, from up to down, are showed in the Fig. 3. 


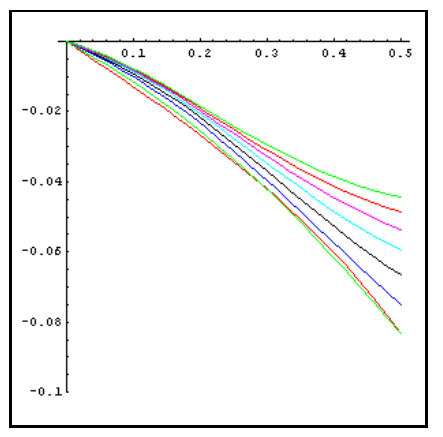

Figure 3: Graphs for the moments $M_{8}^{*}$ to $M_{1}^{*}$

Example 3.14. We showed the graphs for several moments in the example above. In general, the moments $M_{m, n}$ are algebraic functions in $r$. With their aid we can calculate the complex moments $\sigma_{i, j}$. Several of them are as follow:

$\sigma_{2,3}=\frac{1-i}{20} \frac{9-18 r+26 r^{2}}{1-2 r+3 r^{2}}$

$\sigma_{4,3}=\frac{1+i}{280} \frac{164-984 r+3539 r^{2}-7596 r^{3}+10769 r^{4}-8970 r^{5}+3879 r^{6}}{1-6 r+22 r^{2}-48 r^{3}+70 r^{4}-60 r^{5}+27 r^{6}}$

$\sigma_{3,5}=\frac{-i}{28} \frac{26-156 r+554 r^{2}-1176 r^{3}+1640 r^{4}-1344 r^{5}+567 r^{6}}{1-6 r+22 r^{2}-48 r^{3}+70 r^{4}-60 r^{5}+27 r^{6}}$

With these complex moments is possible to calculate the orthogonal polynomial. We give some of them with $r=1 / 4$ :

$p_{0}(z)=1, p_{1}(z)=2.44949((-.5+.5 i)+z)$

$\left.p_{2}(z)=-.3816 i(-i)-2(1-i) z+2 z^{2}\right)$,

$p_{3}(z)=.64523(1+i)-1.95982 i z-.04824(1-i) z^{2}-1.24222 z^{3}$

$p_{4}(z)=4.54935+3.44275 i-(1.47926+8.99667 i) z+(.42282-.2789 i) z^{2}$ $-(1.0742+1.03528 i) z^{3}-2.88825 z^{4}$

$p_{5}(z)=-5.19255-.45243 i+(5.43404+6.23741 i) z-(.15014-.46633 i) z^{2}$

$+(1.34706-.26563 i) z^{3}-(2.24282-1.49228 i) z^{4}-(.81381-.034468 i) z^{5}$

\section{Association (or concordance) measures}

Let us recall that concordance or discordance is fundamental when introducing measures of association. Formally, two ordered pairs of real numbers, $\left(x_{1}, y_{1}\right)$ and $\left(x_{2}, y_{2}\right)$, are concordant if $\left(x_{1}-x_{2}\right)\left(y_{1}-y_{2}\right)>0$. Otherwise they are said to be discordant.

Let $\left(X_{1}, Y_{1}\right)$ and $\left(X_{2}, Y_{2}\right)$ be two continuous random pairs with the same marginal distribution functions, and associated copulas $C_{1}$ and $C_{2}$, respec- 
tively. A concordance function is defined by

$$
Q\left(C_{1}, C_{2}\right)=P\left[\left(X_{1}-X_{2}\right)\left(Y_{1}-Y_{2}\right)>0\right]-P\left[\left(X_{1}-X_{2}\right)\left(Y_{1}-Y_{2}\right)<0\right] .
$$

For a review of concordance measures and the role that copulas play in the study of dependence or association between random variables, see [15, Chap. 5]. Specifically, in the case the case that $H_{1}$ and $H_{2}$ are doubly stochastic measures with the same marginal distribution functions $F$ and $G$, the next result is a main tool for studying the concordance function $Q$ :

Theorem 4.1 ([15, p.159]). Let $\left(X_{1}, Y_{1}\right)$ and $\left(X_{2}, Y_{2}\right)$ be independent vectors of continuous random variables with joint distribution functions $H_{1}$ and $\mathrm{H}_{2}$, respectively, and with common marginals $F$ and $G$. Let $C_{1}$ and $C_{2}$ denote the copulas of $\left(X_{1}, Y_{1}\right)$ and $\left(X_{2}, Y_{2}\right)$, respectively, so that $H_{1}(x, y)=$ $C_{1}(F(x), G(y))$ and $H_{2}(x, y)=C_{2}(F(x), G(y))$. Then

$$
Q=Q\left(C_{1}, C_{2}\right)=4 \int_{\mathbb{I}^{2}} C_{2}(x, y) d \mu_{C_{1}}(x, y)-1 .
$$

We recall three copulas of particular importance: $\Pi(x, y)=x y, M(x, y)=$ $\min (x, y)$ and $W(x, y)=\max (x+y-1,0)$, for all $(x, y) \in \mathbb{I}^{2}$. Moreover, for each copula $C$,

$$
W \leq C \leq M
$$

Now, we study several measures of association which calculate the probability of concordance between random variables with a given copula.

Definition 4.2. Let $(X, Y)$ be a continuous random pair with associated copula $C$. The value $Q(C, C)$ is a measure of association called the Kendall's $\tau$ of $(X, Y)$. Moreover, the value $3 Q(C, \Pi)$ is a measure of association called the Spearman's $\rho$ of $(X, Y)$. And, the value $Q(C, M)+Q(C, W)$ is another measure of association for $(X, Y)$ called the Gini's $\gamma$.

Now, by applying (6) and using (3) and (4), we can express the concordance in terms of the family of copulas $C_{T_{r}}$.

Proposition 4.3. Given the copula $C_{T_{r}}=C_{r}$ with parameter $\left.r \in\right] 0, \frac{1}{2}[$, the following equalities hold:

a) $\int_{\mathbb{I}^{2}} \max (x+y-1,0) d \mu_{C_{r}}(x, y)=\frac{1-r}{8-10 r}$, 
b) $\int_{\mathbb{I}^{2}} \min (x, y) d \mu_{C_{r}}(x, y)=\frac{3-4 r}{8-10 r}$

c) $\int_{\mathbb{I}^{2}} x y d \mu_{C_{r}}(x, y)=1 / 4$,

d) $\int_{\mathbb{I}^{2}} C_{r}(x, y) d \mu_{C_{r}}(x, y)=1 / 4$.

Proof. a) Let us decompose the integral as a sum on five regions in the unit square. To be precise: they are the sets $F_{i}\left(\mathbb{I}^{2}\right)$, with $i=1,2,3,4,5$, where the similarities $F_{i}$ are given by $F_{1}(x, y)=(r x, r y), F_{2}(x, y)=(r x+1-r, r y)$, $F_{3}(x, y)=(r x, r y+1-r), F_{4}(x, y)=(r x+1-r, r y+1-r), F_{5}(x, y)=$ $((1-2 r) x+r,(1-2 r) y+r)$. The decomposition of the unit square, for $r=2$, is showed in Fig. 4.

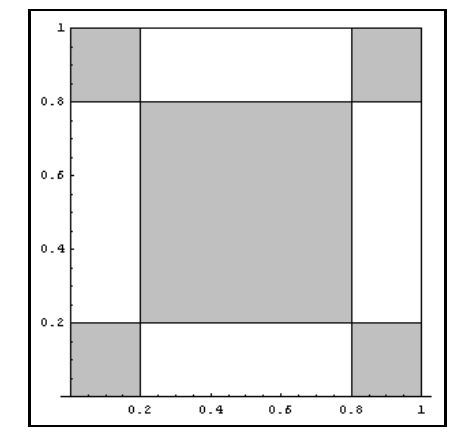

Figure 4: Integration regions 
The measure $\mu_{C_{r}}$ is self-similar, and therefore:

$$
\begin{aligned}
\int_{\mathbb{I}^{2}} W(x, y) d \mu_{C_{r}}(x, y)= & \frac{r}{2} \int_{\mathbb{I}^{2}} W(r x, r y) d \mu_{C_{r}}+\frac{r}{2} \int_{\mathbb{I}^{2}} W(r x+1-r, r y) d \mu_{C_{r}} \\
& +\frac{r}{2} \int_{\mathbb{I}^{2}} W(r x, r y+1-r) d \mu_{C_{r}} \\
& +\frac{r}{2} \int_{\mathbb{I}^{2}} W(r x+1-r, r y+1-r) d \mu_{C_{r}} \\
& +(1-2 r) \int_{\mathbb{I}^{2}} W((1-2 r) x+r,(1-2 r) y+r) d \mu_{C_{r}} \\
= & \frac{r}{2} \int_{\mathbb{I}^{2}} 0 d \mu_{C_{r}}+\frac{r}{2} \int_{\mathbb{I}^{2}} r W(x, y) d \mu_{C_{r}} \\
& +\frac{r}{2} \int_{\mathbb{I}^{2}} r W(x, y) d \mu_{C_{r}}+\frac{r}{2} \int_{\mathbb{I}^{2}}(r x+r y+1-2 r) d \mu_{C_{r}} \\
& +(1-2 r)^{2} \int_{\mathbb{I}^{2}} W(x, y) d \mu_{C_{r}} \\
= & \left(2 r^{2}+(1-2 r)^{2}\right) \int_{\mathbb{I}^{2}} W(x, y) d \mu_{C_{r}}(x, y)+\frac{r(1-2 r)}{2} ;
\end{aligned}
$$

and working out the integral, we have the statement.

b) We proceed as in the above case.

c) It is, in fact, the moment $M_{11}$. 
d) We decompose the integral as in the first case:

$$
\begin{aligned}
\int_{\mathbb{I}^{2}} C_{r}(x, y) d \mu_{C_{r}}(x, y)= & \frac{r}{2} \int_{\mathbb{I}^{2}} C_{r}(r x, r y) d \mu_{C_{r}}+\frac{r}{2} \int_{\mathbb{I}^{2}} C_{r}(r x+1-r, r y) d \mu_{C_{r}} \\
& +\frac{r}{2} \int_{\mathbb{I}^{2}} C_{r}(r x, r y+1-r) d \mu_{C_{r}} \\
& +\frac{r}{2} \int_{\mathbb{I}^{2}} C_{r}(r x+1-r, r y+1-r) d \mu_{C_{r}} \\
& +(1-2 r) \int_{\mathbb{I}^{2}} C_{r}((1-2 r) x+r,(1-2 r) y+r) d \mu_{C_{r}} \\
= & \frac{r}{2} \int_{\mathbb{I}^{2}} \frac{r}{2} C_{r}(x, y) d \mu_{C_{r}}+\frac{r}{2} \int_{\mathbb{I}^{2}} \frac{r}{2} y+\frac{r}{2} C_{r}(x, y) d \mu_{C_{r}} \\
& +\frac{r}{2} \int_{\mathbb{I}^{2}} \frac{r}{2} x+\frac{r}{2} C_{r}(x, y) d \mu_{C_{r}}+\frac{r}{2} \int_{\mathbb{I}^{2}} \frac{r}{2}+1-2 r \\
& +\frac{r}{2}(x+y)+\frac{r}{2} C_{r}(x, y) d \mu_{C_{r}} \\
& +(1-2 r) \int_{\mathbb{I}^{2}} \frac{r}{2}+(1-2 r) C_{r}(x, y) d \mu_{C} \\
= & \left(r^{2}+(1-2 r)^{2}\right) \int_{\mathbb{I}^{2}} C_{r}(x, y) d \mu_{C_{r}}(x, y) \\
& +\frac{3}{4} r^{2}+r(1-2 r),
\end{aligned}
$$

and the result follows.

We conclude by pointing out an interesting and uncommon property concerning the three measures of association defined above. Let us note that a measure of concordance of zero indicates that there is no tendency for one random variable to either increase or decrease when the other increases.

Corollary 4.4. Kendall's $\tau$, Spearman's $\rho$, and Gini's $\gamma$ are zero for all $r \in] 0, \frac{1}{2}[$.

Acknowledgement 4.5. This work has been supported by the Spanish Ministry of Science and Innovation, through projects MTM2011-22394 and TIN201020900-C04-02, and by ERDF-FEDER funds.

\section{Appendix}

In this section we show the Java implementation for computing the moments described in corollaries 3.5, 3.9 and 3.12. Implementation of Corollary 
3.5 is shown in Fig. 5. Figure 6 displays the implemention of Corollary 3.9 whilst the Java code for Corollary 3.12 can be found in Fig. 7. The methods in Fig. 8 are just instrumental, and they are used in the other three implementations.

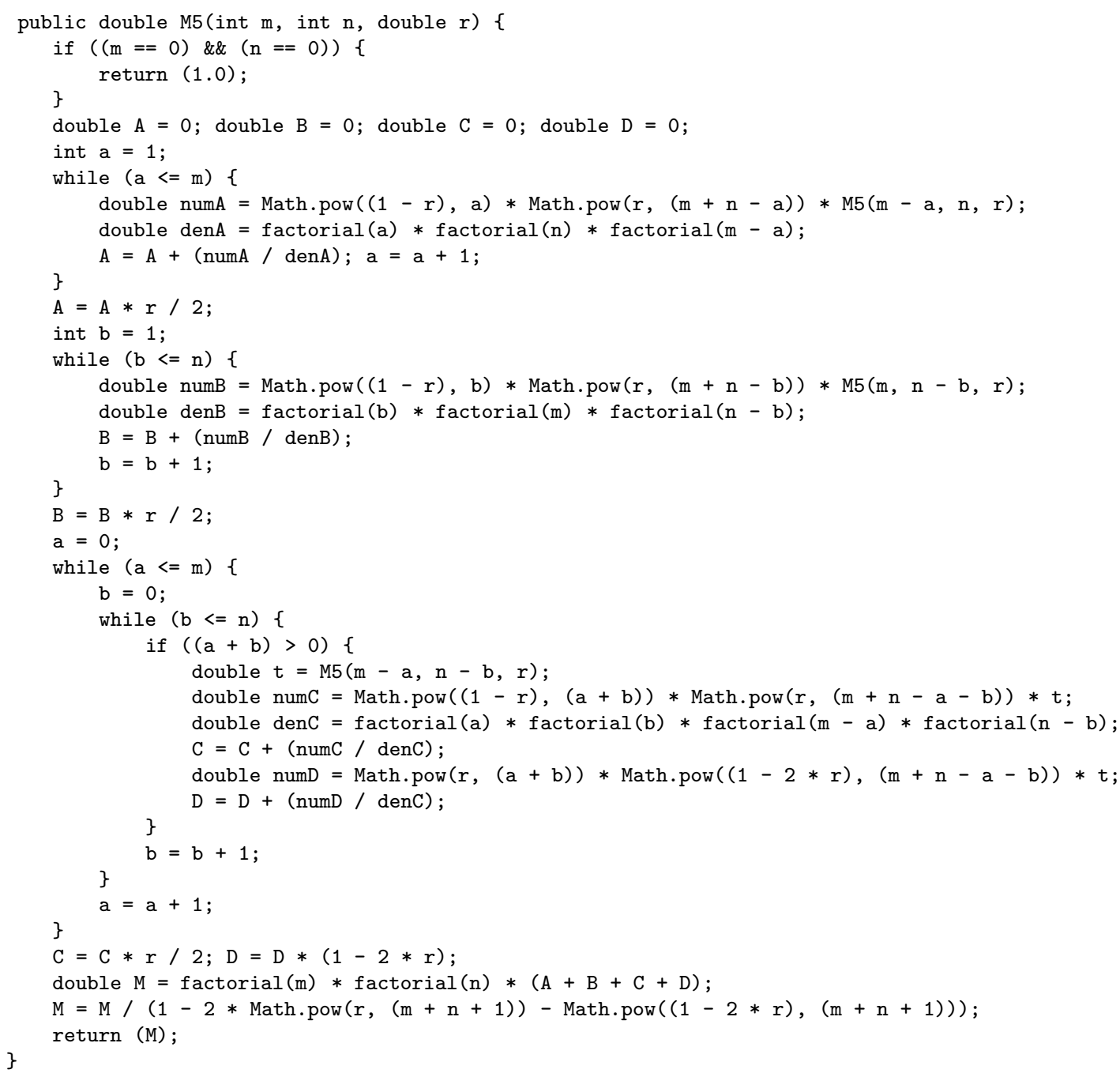

Figure 5: Java implementation of the moment in Corollary 3.5. 


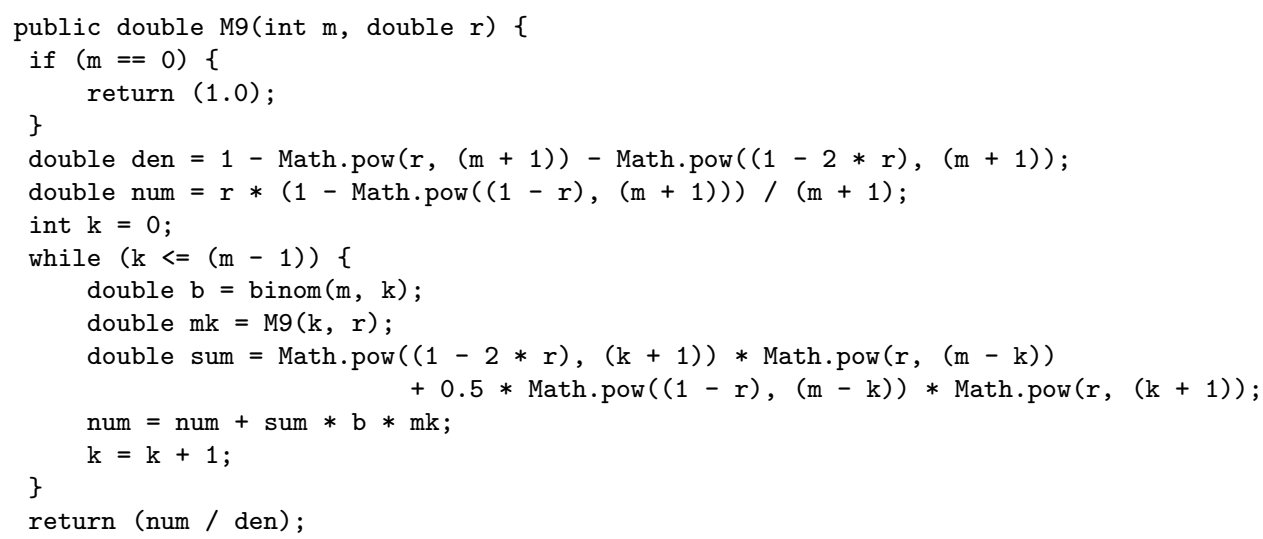

Figure 6: Java implementation of the moment in Corollary 3.9.

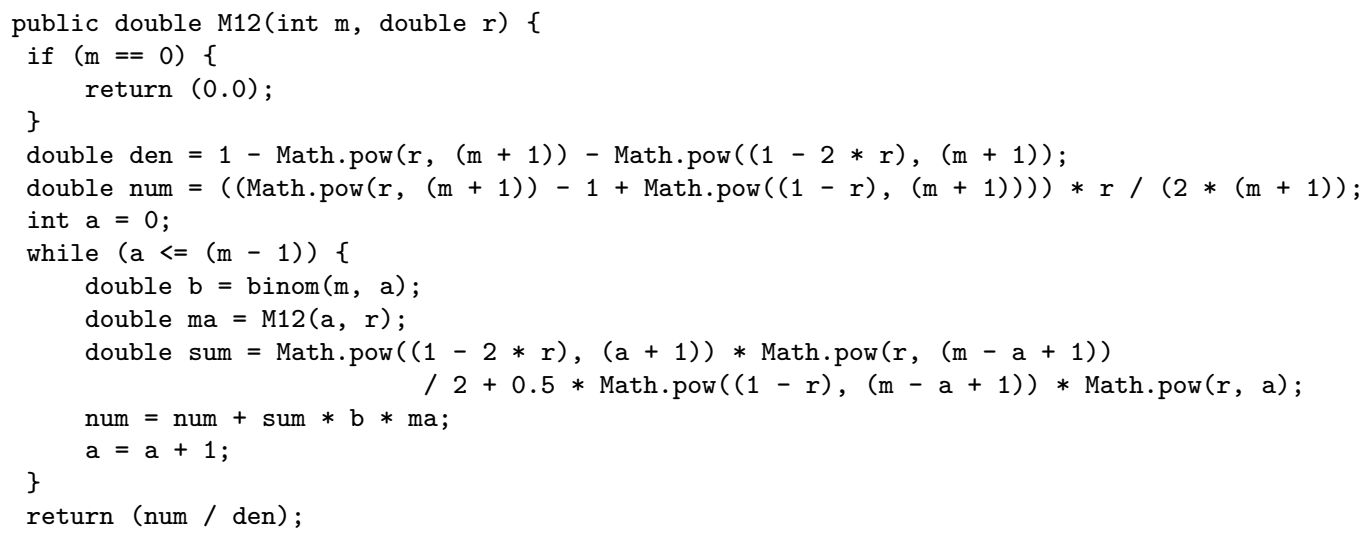

Figure 7: Java implementation of the moment in Corollary 3.12 . 


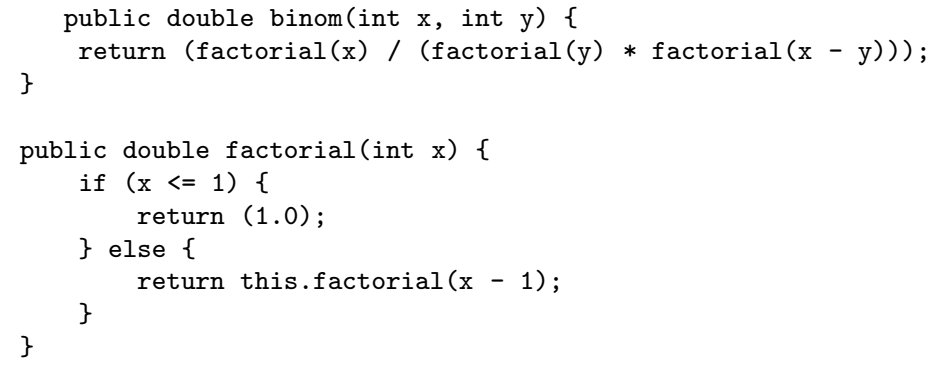

Figure 8: Technical methods used in the implementations of the moments.

[1] de Amo, E., Díaz Carrillo, M. and Fernández-Sánchez, J.: Copulas and associated fractal sets, J. Math. Anal. Appl. 386 (2012) 528-541

[2] de Amo, E., Díaz Carrillo, M. and Fernández-Sánchez, J.: Singular Functions with Applications to Fractals and Generalised Takagi's Functions, To appear in Acta Appl. Math. (2012). DOI 10.1007/s10440-0119665-Z

[3] Baek, I.-S.: A Note on the moments of the Riesz-Nágy-Takcs distribution, J. Math. Anal. Appl. 348 (2008) 165-168

[4] Billingsley, P.: Probability and measure (3rd ed.), Wiley Series in Probability and Mathematical Statistics. John Wiley \& Sons, Inc., New York, 1995

[5] Cartan, H.: Elementary Theory of Analytic Functions of One or Several Complex Variables, Addison-Wesley, 1963

[6] Fredricks, G.A., Nelsen, R.B. and Rodríguez-Lallena, J.A.: Copulas with fractal supports, Insurance Math. Econom. 37 (2005) 42-48

[7] Edgar, G.A.: Measure, Topology, and Fractal Geometry, Undergraduate Texts in Mathematics, Springer, Berlin, Heidelberg, New York, 1990

[8] Falconer, K.J.: Fractal Geometry: Mathematical Foundations and Applications (2nd ed.), John Wiley \& Sons, 2003

[9] Falconer, K.J.: Techniques in Fractal Geometry, John Wiley \&Sons, 1997 
[10] Goh, W. and J. Wimp, J.: Asymptotics for the moments of singular distributions, J. Approx. Theory 74 (1993) 301-334

[11] Goh, W. and J. Wimp,J.: A generalized Cantor Riesz-Nágy function and the growth of its moments, Asymptot. Anal. 8 (1994) 379-392

[12] Hutchinson, J.E.: Fractals and self-similarity, Indiana Univ. Math. J. 30 (5) (1981) 713-747

[13] Lad, F.R. and Taylor, W.F.C.: The moments of the Cantor distribution, Statist. Probab. Lett. 13 (1992) 307-310

[14] McClure, M: The Hausdorff dimension of Hilbert's coordinate functions, The Real Analysis Exchange 24 (1998/99) 875-883

[15] Nelsen, R.B.: An introduction to copulas (2nd ed.) Springer Series in Statistics, New York, 2006

[16] de Rham, G.: Sur certaines equations fonctionnelles, Ecole Polytechnique de l'Universite de Lausanne, Centenaire 1853-1953. Lausanne (1953) 95-97

[17] Rudin, W.: Real and Complex Analysis, McGraw-Hill, New York, 1966

[18] Sklar, A.: Fonctions de répartition à $n$ dimensions et leurs marges, Publ. Inst. Statidt. Univ. Paris 8 (1959) 229-231

[19] Sagan, H.: Space Filling Curves, Springer-Verlag, New York, 1994

[20] Stahl, H. and Totik, V.: General orthogonal polynomials, 43 Cambridge University Press, Cambridge, 1992

[21] Szego, G.: Orthogonal Polynomials, AMS Colloquium Publ. vol. 23, 1939

[22] Urbański, M.: The Hausdorff dimension of the graphs of continuous selfaffine functions, Proc. Amer. Math. Soc. 108 (4) (1990) 921-930 